\title{
THE RELATIONSHIP BETWEEN THE PHOTOSYNTHETIC PIGMENTS, CAROTENOIDS AND YIELD OF BROOMCORN MILLET (PANICUM MILIACEUM; POACEAE)
}

\author{
LI, M. ${ }^{1}$ - WANG, Z. ${ }^{1}-$ CHEN, L. Q. ${ }^{1}-$ WANG, J. J. ${ }^{1}-$ LI, H. Y. ${ }^{1}-$ HAN, Y. H. ${ }^{1,2,3}-$ ZHANG, B. ${ }^{1,2,3 *}$ \\ ${ }^{1}$ College of Agricultural, Shanxi Agricultural University, Taigu, Shanxi 030801, People's \\ Republic of China
}

${ }^{2}$ Institute of Agricultural Bioengineering, Shanxi Agricultural University, Taigu, Shanxi 030801, People's Republic of China

${ }^{3}$ Shanxi Key Laboratory of Resources and Genetic Improvement of Minor Crops, Taigu 030801, People's Republic of China

${ }^{*}$ Corresponding author

e-mail: Abingood@126.com

(Received 24 ${ }^{\text {th }}$ Jul 2020; accepted $22^{\text {nd }}$ Oct 2020)

\begin{abstract}
With advantages, such as high efficiency of water use and drought-resistance, broomcorn millet (Panicum miliaceum; Poaceae) will be a potential crop to be developed in the future. Finding methods to achieve high yield and good quality for broomcorn millet is essential to relieve the pressure of growing population and deteriorating environment. Since photosynthetic pigments play important roles in photosynthesis and the blooming stage is a vital stage in determining yield, at this stage, we measured photosynthetic pigment contents of five varieties and analyzed the relationship between photosynthetic pigment contents, photosynthesis and yield. The results showed that photosynthetic pigment contents could influence the photosynthetic efficiency of broomcorn millet and there was a positive relationship between that and crop yield. But there was no direct relationship between that and crop yield, which could make it clear that photosynthetic pigment contents influence the yield by regulating photosynthesis. Besides, healthy and nutritious food has been sought by people. As a kind of healthy food, we analyzed the carotenoid contents in broomcorn millet and explored the relationship between yield and carotenoid contents. We found no significant correlation between qualities and yield, which provide a new idea that we can enhance yield and quality at the same time.
\end{abstract}

Keywords: broomcorn millet, photosynthetic pigment, photosynthesis, kernel traits, carotenoids, beige

\section{Introduction}

Broomcorn millet is one of the oldest cultivated cereal, which has existed for 10000 years (Lu et al., 2009). As an extensively cultivated crop, it has the lowest requirements compared with many major cereals in water availability. Besides, it has other advantages, such as, high efficiency of water use (Diao, 2017; Hou et al., 2017), high drought-resistance, C4 photosynthetic pathway (Pinto et al., 2014; Xue et al., 2019) short growing season, heat resistance and superficial root system (Amadou et al., 2013; Habiyaremye et al., 2017). With the population growing rapidly and the environment deteriorating gradually, there is an augment in food demand (Challinor et al., 2014; Leipe et al., 2019). Confronted with the challenge in food security and environmental pollution, broomcorn millet may arise to be the greatest potential crop in the development of agriculture in the future. In addition, broomcorn millet is of great nutritious and its edible value (Habiyaremye et al., 2017; Azad et al., 2019), medicinal 
value and energy value (Zhang et al., 2014; Dong et al., 2019) have also been gradually recognized and exploited.

The continuous decline of cultivated land, the increasing population and climate warming (Princiotta et al., 2014) have attracted people's attention to crop yield for a long time. The yield of crop is influenced by its internal factors and external factors, including genotype, agronomic characters, photosynthesis, water content, temperature and climate. Among those factors, photosynthetic efficiency is an important one, and it has a positive influence on the formation of yield. Then a unique $\mathrm{C} 4$ photosynthetic pathway has arisen to be a research hotspot in recent studies. Most studies have shown that $\mathrm{C} 4$ plants have a high photosynthetic rate, and the photosynthesis of $\mathrm{C} 4$ can improve the water use efficiency of plants. As a $\mathrm{C} 4$ plant, broomcorn millet is one of the favorite cereals because of its high drought resistance, high photosynthetic efficiency and high efficiency of water use. In addition, although most studies have been done to explore regulation enzymes in $\mathrm{C} 4$ photosynthetic pathway (Wang et al., 2014; Shi et al., 2020), almost no study has been made to explore the photosynthesis by analyzing physiological indices, such as the relationship between photosynthetic efficiency and photosynthetic pigment contents. It is well known that photosynthetic pigments, especially chlorophyll, plays a key role in the photosynthesis, and based on this idea, we intended to learn whether there is a direct relationship between them.

Besides, people have paid more and more attention to the nutrition and health of crops, and thus it is rather important to improve crop quality. Research shows that carotenoids are antioxidants and can prevent various eye and cardiovascular diseases. Broomcorn millet is rich in carotenoids. However, most tests showed that the yield of crop contradicts crop quality. So we intended to analyze whether high yield and high carotenoid contents can coexist in broomcorn millet, and provide a basis for further study of broomcorn millet yield and quality.

\section{Materials and methods}

\section{Plant material}

All broomcorn millet varieties, including Jinshu 2 (JS2), Jinshu 5 (JS5), Jinshu 7 (JS7), Nongjiazhong (NJZ) and Yidianhong (YDH), are from Shanxi Province, China. The varieties with good characters and qualities were selected in this study. All broomcorn millet varieties were sown on the $4^{\text {th }}$ May, 2017 at the field research station in Shanxi Agricultural University. The area is located at $112^{\circ} 28^{\prime} \mathrm{N}, 37^{\circ} 12^{\prime} \mathrm{E}$ belonging to temperate continental climate, with an altitude of $803 \mathrm{~m}$, annual rainfall of about $462.9 \mathrm{~mm}$, average annual temperature of $9.9^{\circ} \mathrm{C}$ and frost-free period of about $176 \mathrm{~d}$. Each variety is planted in a plot with an area of $50 \mathrm{~m}^{2}(5 \times 10)$. The sowing time of broomcorn millet in spring is generally in the middle and late May. The sowing depth of broomcorn millet is $3 \sim 5 \mathrm{~cm}$. According to the soil moisture content, sowing can be done in advance or later, shallow sowing should be done when the soil moisture content is good, deep sowing should be done when the soil moisture content is poor, and moderate suppression should be done, and attention should be paid to taking advantage of the rain to rob the soil moisture for sowing. Water management: Irrigation was carried out twice in the whole growth period, once in the jointing stage (10th June) and once in the filling stage (20th July). Other cultivation measures are the same as those of general field production management. We made the following experimental design for five varieties of broomcorn millet (Fig. 1). 


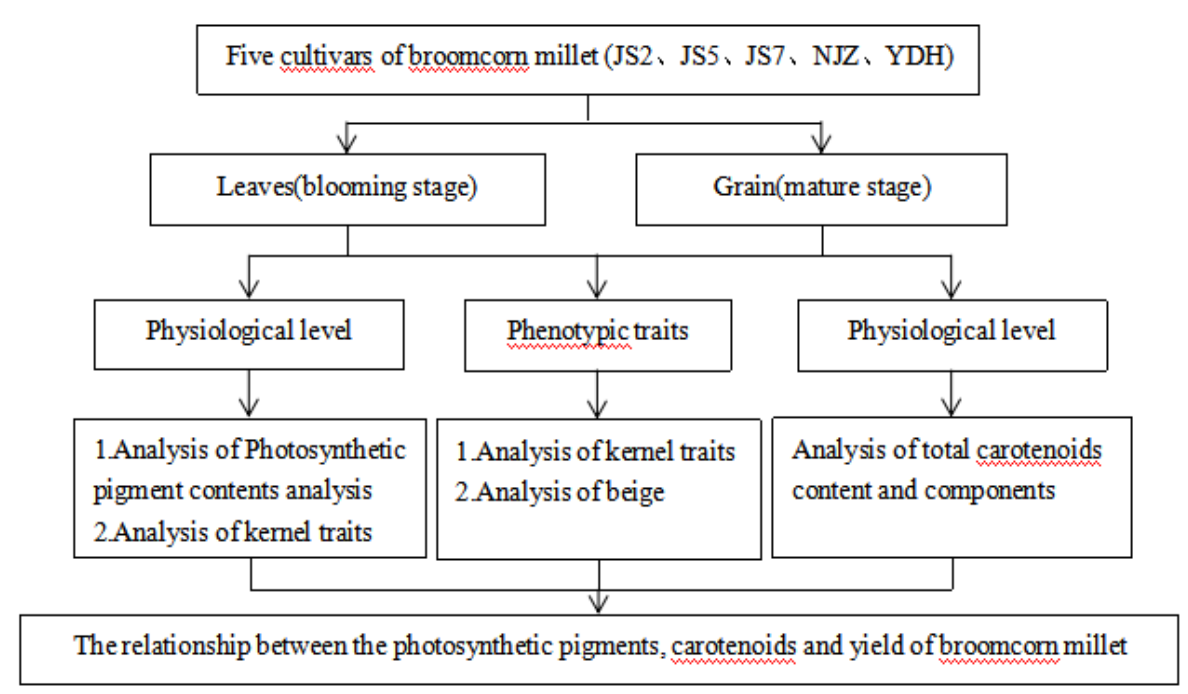

Figure 1. Experimental design for five varities of broomcorn millet

\section{Photosynthetic pigment contents analysis}

The photosynthetic pigment contents of five broomcorn millet varieties were measured at the blooming stage ( $30^{\text {th }}$ July). The reciprocal first leaf of three healthy and consistent plants was selected for each variety, each of which was taken from the middle of the leaf to remove the midvein, and grind it with liquid nitrogen. Every sample $(0.2 \mathrm{~g}$ for each of the three duplicates for each variety) was transferred to a new $10 \mathrm{~mL}$ centrifuge tube and mixed with $10 \mathrm{~mL}$ solution of $95 \%$ ethyl alcohol, then extracted in an oscillator for $24 \mathrm{~h}$ until the tissue faded. Centrifuged and measured. The content of photosynthetic pigments was determined at the wavelength of $470 \mathrm{~nm}$, $665 \mathrm{~nm}$ and $649 \mathrm{~nm}$ with 95\% ethanol as blank (Eqs.1-4) (Rasool et al., 2020).

$$
\begin{gathered}
\text { Chla }=\frac{\left(13.95 \times A_{665}-6.88 \times A_{649}\right) \times 0.01}{0.2} \\
\text { Chlb }=\frac{\left(24.96 \times A_{649}-7.32 \times A_{665}\right) \times 0.01}{0.2} \\
\text { Caro }=\frac{\left(1000 \times A_{470}-7.32 \times \text { Chla }-104 \times \text { Chlb }\right) \times 0.01}{0.2 \times 229} \\
\text { Chl }=\text { Chla }+ \text { Chlb }
\end{gathered}
$$

\section{Analysis of photosynthetic indices}

Three plants in the same growth were measured by using the LI-6400 portable photosynthesis analyzer in each variety. Photosynthetic indices and chlorophyll fluorescence kinetic parameters of reciprocal first leaf were analyzed at 8:30 on a sunny morning. Photosynthetic indices include Net Photosynthetic Rate (Photo), Transpiration Rate (Trmmol) and Stomatal Conductance (Cond) (Huang et al., 2016). 


\section{Analysis of kernel traits}

Three kernel traits, including spike weight per plant (SWPP), grain weight per plant (GWP) and weight of 1000 grain (KGW), were analyzed with five varieties at the mature stage, and five plants were measured in every variety.

\section{Analysis of total carotenoids}

Millet ( $0.6 \mathrm{~g}$ for each of the three duplicates for each variety) was ground into powder, transferred to a $10 \mathrm{~mL}$ centrifuge tube, and mixed with $6 \mathrm{~mL}$ water-saturated n-butanol fully. After shaken about $3 \mathrm{~h}$ in room temperature, centrifuged at $8000 \mathrm{r} / \mathrm{min}$ for $10 \mathrm{~min} .0 .6 \mathrm{ml}$ supernatant was transferred to a new centrifuge tube to have a high-performance liquid chromatography (HPLC) analysis. The surplus supernatant was to measure by ultraviolet-visible spectrophotometer (MAPADA UV-1200 type), and the extract was measured at $450 \mathrm{~nm}$. The whole experiment was carried out under dark conditions due to the easy decomposition of carotenoids. Total carotenoid content (Eq.5) was calculated according to the formula, in which A represents the absorption value at $450 \mathrm{~nm}$ wavelength; $\mathrm{V}$ represents the extraction volume $(\mathrm{mL}) ; \mathrm{m}$ represents the sample mass (g); and 0.250 represents the conversion coefficient (Dong et al., 2014).

$$
\text { Total carotenoid content }=\frac{\frac{A}{0.25} \times V}{m}
$$

\section{Analysis of high-performance liquid chromatography (HPLC)}

By using the chromatographic instrument (Liu et al., 2016), carotenoids were separated by C30 column $(250 \mathrm{~mm} \times 4.6 \mathrm{~mm}, 5 \mu \mathrm{m}$, YMC Company, Japan); with mobile phase A, methanol : methyl tert-butyl ether (MTBE) : $\mathrm{H}_{2} \mathrm{O}(81: 15: 4$, V/V/V) and mobile phase B, MTBE : methanol (90:10,V/V); with grads elution; at a flow rate of 1 $\mathrm{mL} / \mathrm{min}$; monitoring wavelength was $450 \mathrm{~nm}$; injection volume was $20 \mathrm{uL}$; column temperature was $35{ }^{\circ} \mathrm{C}$. Lutein and zeaxanthin were thoroughly separated with the average retention time of $9 \mathrm{~min}$ and $10 \mathrm{~min}$, respectively. $\mathrm{X}$ represents the concentration of injection volume $(\mu \mathrm{g} / \mathrm{mL}), Y$ represents peak area $(\mathrm{mAU} \cdot \mathrm{min})$. The regression equation of lutein and zeaxanthin were obtained by analysis (Sigma company) (Eqs.6-7).

$$
\begin{gathered}
\text { Lutein }: Y=2.2707 X\left(R^{2}=0.9991\right) \\
\text { Zeaxanthin }: Y=2.5785 X\left(R^{2}=0.99932\right)
\end{gathered}
$$

\section{Beige analysis}

After husking the broomcorn millet, the color of millets was determined with a noncontact colorimeter (X-Rite VS450 colorimeter). Through using CIE L ${ }^{*}{ }^{*} b^{*}$ colorimeter system, determination of index $\mathrm{L}^{*}, \mathrm{a}^{*}, \mathrm{~b}^{*}$ and the orange index of CCI was calculated (Eq.8), where $\mathrm{L}^{*}$ denotes the brightness; a* shows the level of red / green (red), and the positive numbers represent reddish and negative numbers represent greenish color, the greater the absolute is, the darker the color will be. $b^{*}$ shows yellow / blue degree, and positive numbers express yellow, and negative bias blue, the greater the absolute value is, the darker of color will be. Positive CCI expresses red, a negative value indicates the blue 
and green, and 0 presents the mixed color in red, yellow, blue and green (Zhou et al., 2010; Fu et al., 2012). Each species was set up with 3 biological repeats for beige determination.

$$
C C I=\frac{1000 \times a^{*}}{L^{*} \times b^{*}}
$$

\section{Data analysis}

All figures and tables were finished by using Excel 2003. One-way analysis of variance (ANOVA), the Duncan's multiple range tests and Pearson correlation tests were used to assess each of the parameters through SPSS statistics software (Version 19, SPSS, Chicago, IL, United States).

\section{Results}

\section{Photosynthetic pigment contents analysis at the blooming stage}

Photosynthetic pigment contents, including Chlorophyll A (Chla), Chlorophyll B (Chlb), Carotenoid (Caro) and total Chlorophyll contents (Total CHL), were analyzed during the blooming stage $\left(30^{\text {th }}\right.$ July). Except for obvious aggrandizement in Chlb content in YDH (Fig. 2B), each variety had the same position in different photosynthetic pigment contents, and various photosynthetic pigment contents in JS7 were maximum and JS5 had minimum contents among all measured varieties. Then the analysis of photosynthetic pigment contents exhibited a slight difference in JS2 and NJZ, but no significant difference was found in two varieties (Fig. 2).

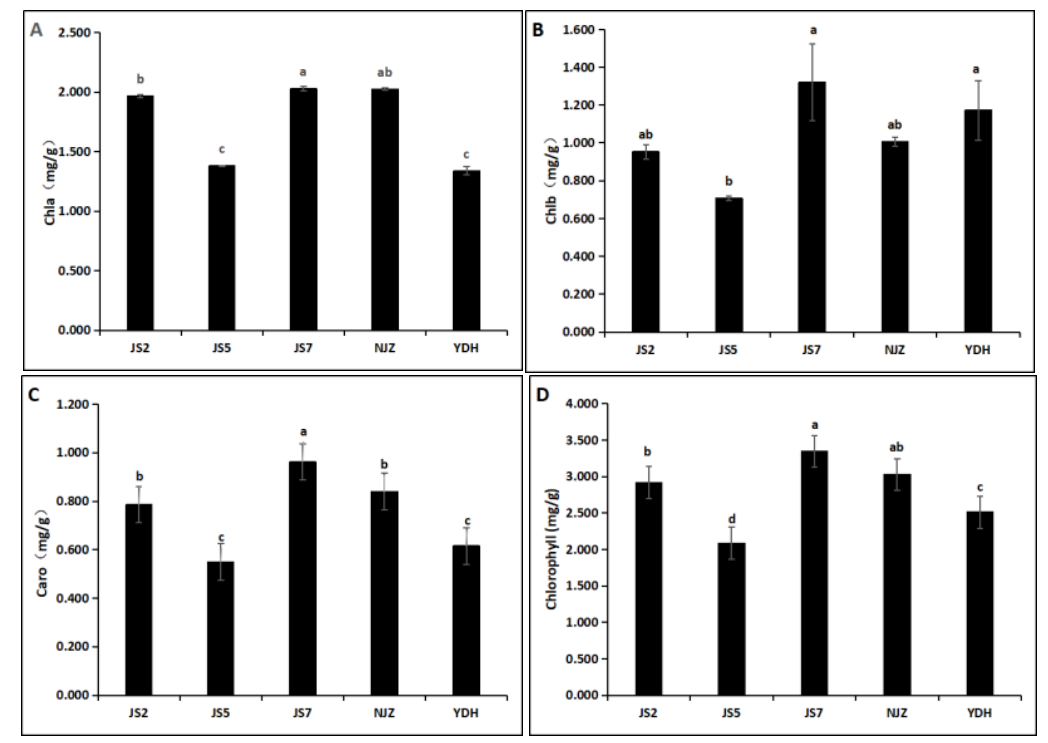

Figure 2. Photosynthetic pigment contents analysis with five varieties at the blooming stage. (A) Chlorophyll A (Chla) contents of five varieties. (B) Chlorophyll B (Chlb) contents of five varieties. (C) Carotenoid (Caro) contents of five varieties. (D) Chlorophyll contents of five varieties. Note: The error bars indicate standard error. Different lowercase letters indicate significant difference at $P<0.05$ 


\section{Photosynthetic indices analysis of five varieties at the blooming stage}

Photosynthetic indices, including Net Photosynthetic Rate (Photo), Transpiration Rate (Trmmol), and Stomatal Conductance (Cond), were measured. Three plants with the same growth were measured by using the LI-6400 portable photosynthesis analyzer in each variety, respectively. Photosynthetic indices of reciprocal first leaf were analyzed at 8:30 on a sunny morning. The data revealed there were some distinctions in all varieties with three photosynthetic indices. Obviously, JS5 and YDH were different from other varieties, and JS7 had a maximum value in Photo (Fig. 3A-1). Then no marked difference was found between JS2 and YDH in Trmmol (Fig. 3A-3), and they significantly differed from other varieties in Trmmol and Cond (Fig. 3A-2, Fig. 3A-3). However, the data of Cond exhibited an obvious distinction between JS2 and YDH in Cond (Fig. 3A-2).
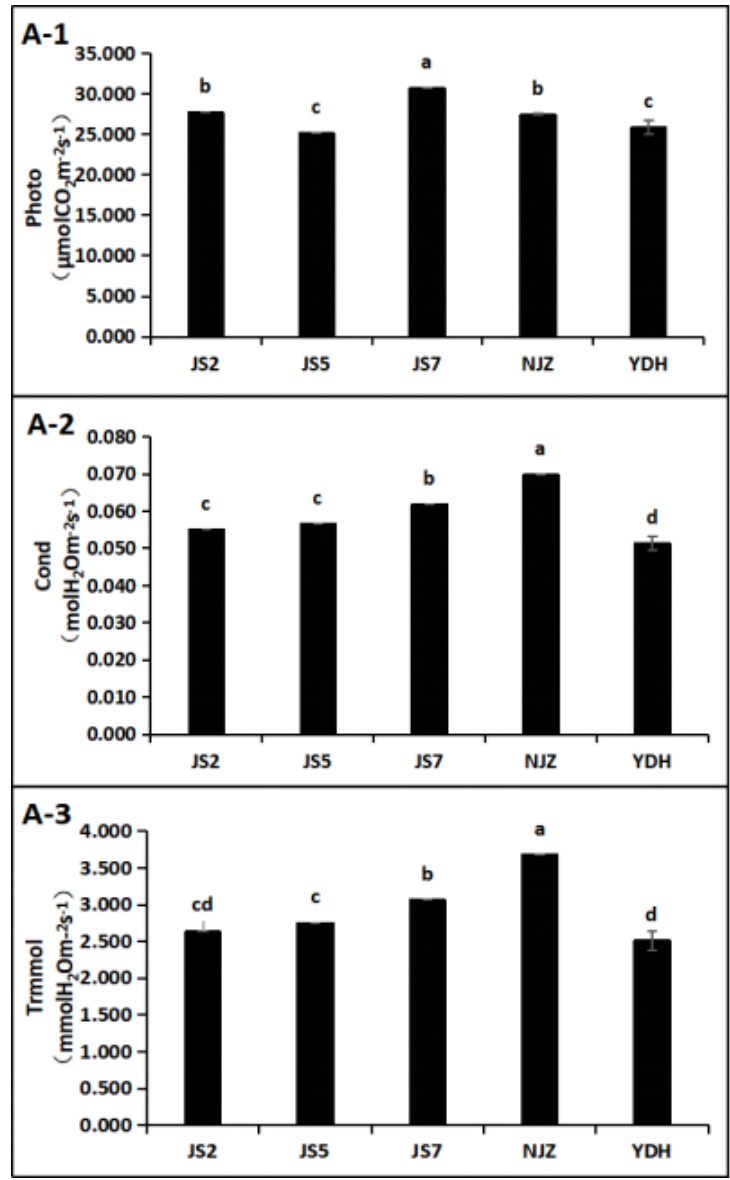

Figure 3. Photosynthetic indices analysis of five varieties in the blooming stage. (A-1) Net Photosynthetic Rate (Photo) of five varieties. (A-2) Stomatal Conductance (Cond) of five varieties. (A-3) Transpiration Rate (Trmmol) of five varieties. Note: The error bars indicate standard error. Different lowercase letters indicate significant difference at $P<0.05$

\section{Correlation analysis between photosynthetic pigment contents and photosynthetic indices at the blooming stage}

Photosynthetic pigments occupied an important position in the photosynthesis of plants, and Chlorophyll played a main role in these pigments. To investigate whether 
there was a correlation between photosynthetic pigment contents and photosynthetic indices, a correlation analysis was done (Table 1). Except for Chlb, a significant correlation was detected between photosynthetic pigment contents and Photo. Besides, an obvious correlation was revealed with each other in the photosynthetic pigment contents, but Chla was not markedly correlated with Chlb.

Table 1. Correlation analysis between photosynthetic pigment contents and Photo

\begin{tabular}{c|c|c|c|c}
\hline Pearson & Chla & Chlb & Caro & Photo \\
\hline Chla & 1 & & & \\
Chlb & 0.266 & 1 & & \\
Caro & $.881^{* *}$ & $.652^{* *}$ & 1 & \\
Photo & $.794^{* *}$ & 0.466 & $.868^{* *}$ & 1 \\
\hline
\end{tabular}

“**” means significant correlation at $P<0.01$

\section{Comparison of three kernel traits with five varieties at the mature stage}

Most traits could influence the formation of yield and there was a direct correlation between kernel traits (spike weight per plant, grain weight per plant and weight of 1000 grain) and yield, so kernel traits were important to reflect the yield of crop. Five varieties were investigated in the mature stage and the result revealed that some distinctions were found in three kernel traits with five varieties. Then JS7 had a significantly higher yield than other varieties and YDH had a lower yield than others. Comparing GWP, the KGW of all varieties had a reduction, but YDH was different from them and it showed an obvious increase (Fig. 4).

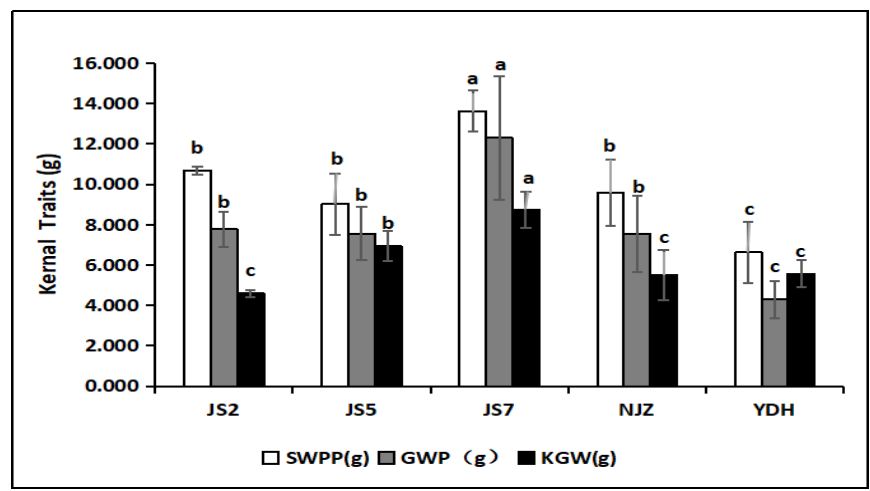

Figure 4. Three kernel traits comparison with five varieties in the mature stage. SWPP: spike weight per plant; GWP: grain weight per plant; KGW: weight of 1000 grain. Note: The error bars indicate standard error. Different lowercase letters indicate significant difference at

$$
P<0.05
$$

\section{Correlation analysis between three kernel traits comparison and photosynthetic indices at the blooming stage}

Photosynthesis has been reported to play a crucial role in the formation of yield, so the correlation was analyzed between three kernel traits and photosynthetic indices. The analysis showed there was a marked correlation between Photo and SWPP. Besides, a 
very significant correlation was detected between GWP and SWPP, Cond and Trmmol, but no significant correlation was found between GWP and KGW, KGW and SWPP (Table 2).

Table 2. Correlation analysis between three kernel traits and photosynthetic indices

\begin{tabular}{c|c|c|c|c|c|c}
\hline Pearson & Photo & Cond & Trmmol & SWPP & GWP & KGW \\
\hline Photo & 1 & & & & & \\
Cond & 0.416 & 1 & & & & \\
Trmmol & 0.357 & $.989^{* *}$ & 1 & & \\
SWPP & $.887 *$ & 0.436 & 0.325 & 1 & & \\
GWP & 0.843 & 0.469 & 0.358 & $.978^{* *}$ & 1 & \\
KGW & 0.515 & 0.215 & 0.145 & 0.597 & 0.74 & 1 \\
\hline \\
“**” means significant correlation at $P<0.01$; “*” means significant correlation at $P<0.05$, the same as \\
below
\end{tabular}

\section{Correlation analysis between three kernel traits comparison and photosynthetic pigment contents in the blooming stage}

By this investigation, we found that photosynthetic pigment contents can directly influence the photosynthesis, and the photosynthesis played a crucial role in the formation of yield. To find out whether photosynthetic pigment directly affects crop yield analysis was done between photosynthetic pigment contents and three kernel traits (Table 3).

Table 3. Correlation analysis between three kernel traits comparison and photosynthetic pigment contents

\begin{tabular}{c|c|c|c|c|c|c}
\hline & SWPP & GWP & KGW & Chla & Chlb & Caro \\
\hline SWPP & 1 & & & & & \\
GWP & $.978^{* *}$ & 1 & & & & \\
KGW & 0.597 & 0.74 & 1 & & & \\
Chla & 0.769 & 0.667 & 0.071 & 1 & & \\
Chlb & 0.356 & 0.336 & 0.368 & 0.359 & 1 & \\
CAR & 0.821 & 0.757 & 0.33 & $.927^{*}$ & 0.654 & 1 \\
\hline
\end{tabular}

“**” means significant correlation at $P<0.01$; “*” means significant correlation at $P<0.05$, the same as below

\section{Extract and analysis of carotenoid and beige in five variety seeds}

Carotenoids have a maximum absorption peak at $450 \mathrm{~nm}$, so total carotenoid contents were measured at $450 \mathrm{~nm}$, and the difference in every component of carotenoid was analyzed. On the whole, lutein and zeaxanthin were major components and the accumulation of lutein was greater than that of zeaxanthin. Some distinctions can be seen in every index among all varieties. JS5 had a maximum accumulation in lutein, zeaxanthin and carotenoid, while JS7 had a minimum accumulation in these components (Table 4). 
Table 4. Carotenoid content analysis of five variety grains in the mature stage

\begin{tabular}{c|c|c|c|c}
\hline & TCC $(\boldsymbol{\mu g} / \mathbf{g})$ & $\mathbf{L C}(\boldsymbol{\mu g} / \mathbf{g})$ & $\mathbf{Z C}(\boldsymbol{\mu g} / \mathbf{g})$ & $(\mathbf{L C}+\mathbf{Z C}) / \mathbf{T C C}(\boldsymbol{\%})$ \\
\hline JS2 & $11.720 \pm 0.040 \mathrm{c}$ & $8.522 \pm 6.026 \mathrm{~b}$ & $2.552 \pm 1.804 \mathrm{~b}$ & 94.484 \\
JS5 & $16.133 \pm 0.589 \mathrm{a}$ & $12.371 \pm 8.747 \mathrm{a}$ & $3.095 \pm 2.188 \mathrm{a}$ & 95.860 \\
JS7 & $11.720 \pm 0.069 \mathrm{c}$ & $8.103 \pm 5.730 \mathrm{~b}$ & $2.207 \pm 1.560 \mathrm{~b}$ & 87.969 \\
NJZ & $15.440 \pm 0.101 \mathrm{a}$ & $11.855 \pm 8.383 \mathrm{a}$ & $2.924 \pm 2.068 \mathrm{a}$ & 95.723 \\
YDH & $12.880 \pm 0.040 \mathrm{~b}$ & $9.339 \pm 6.603 \mathrm{~b}$ & $2.492 \pm 1.762 \mathrm{~b}$ & 91.850 \\
\hline
\end{tabular}

TCC: Total Carotenoids; LC: Lutein; ZC: Zeaxanthin. Different lowercase letters in the same column indicate significant difference at $P<0.05$

The color is a vital factor when people purchase crop, so colors of millets were determined with using the non-contact colorimeter (X-Rite VS450 colorimeter). Through analyzing the beige indices, the difference of millet color in all varieties could be seen. Compared with other varieties, the brightness of JS2 and JS7 had a remarkable difference. Then $a^{*}$ and $b^{*}$ of JS2 were the least bright and significantly differed in all varieties. Except for JS7, the result of CCI showed a slight but not significant distinction in all materials (Table 5).

Table 5. Beige of millet analysis of five variety seeds at the mature stage

\begin{tabular}{c|c|c|c|c}
\hline & $\mathbf{L}^{*}$ & $\mathbf{a}^{*}$ & $\mathbf{b}^{*}$ & CCI \\
\hline JS2 & $62.766 \pm 0.286 \mathrm{~b}$ & $6.546 \pm 0.083 \mathrm{c}$ & $39.988 \pm 0.396 \mathrm{~d}$ & $2.609 \pm 0.035 \mathrm{~b}$ \\
JS5 & $66.994 \pm 0.149 \mathrm{a}$ & $7.444 \pm 0.148 \mathrm{~b}$ & $42.804 \pm 0.572 \mathrm{bc}$ & $2.596 \pm 0.455 \mathrm{~b}$ \\
JS7 & $63.174 \pm 0.254 \mathrm{~b}$ & $7.868 \pm 0.096 \mathrm{a}$ & $43.504 \pm 0.383 \mathrm{ab}$ & $2.864 \pm 0.043 \mathrm{a}$ \\
NJZ & $67.550 \pm 0.249 \mathrm{a}$ & $7.84 \pm 0.086 \mathrm{a}$ & $44.122 \pm 0.237 \mathrm{a}$ & $2.631 \pm 0.038 \mathrm{~b}$ \\
YDH & $67.242 \pm 0.303 \mathrm{a}$ & $7.182 \pm 0.136 \mathrm{~b}$ & $42.156 \pm 0.452 \mathrm{c}$ & $2.533 \pm 0.035 \mathrm{~b}$ \\
\hline
\end{tabular}

Different lowercase letters in the same column indicate significant difference at $P<0.05$

\section{Correlation analysis between carotenoid contents and kernel traits}

The correlation between carotenoid contents and kernel traits was analyzed (Table 6). An obviously positive correlation could be seen in different carotenoid contents and a negative correlation was found between carotenoid contents and kernel traits, although the correlation was not significant.

Table 6. Correlation analysis between carotenoid contents and kernel traits

\begin{tabular}{c|c|c|c|c|c|c}
\hline & SWPP & GWP & KGW & TCC & LC & ZC \\
\hline SWPP & 1 & & & & & \\
GWP & $.978^{* *}$ & 1 & & & & \\
KGW & 0.597 & 0.74 & 1 & & & \\
TCC & -0.419 & -0.293 & -0.052 & 1 & & \\
LC & -0.437 & -0.326 & -0.124 & $.997^{* *}$ & 1 & \\
ZC & -0.466 & -0.399 & -0.319 & $.933^{*}$ & $.956^{*}$ & 1 \\
\hline
\end{tabular}

“**” means significant correlation at $P<0.01$; “*” means significant correlation at $P<0.05$ 


\section{Discussion}

\section{Relationship between photosynthetic pigment contents and photosynthetic indices}

Chlorophyll is a photosynthetic pigment to participate in the absorption, transfer and transformation of light energy (Soares et al., 2020). In addition, it is a significant deciding factor in the efficiency of photosynthesis (Kumar et al., 2020). Through this experiment, we intended to explore whether there was a relationship between photosynthetic indices and photosynthetic pigments. The analysis results about photosynthetic indices and photosynthetic pigment contents showed they were corresponding to each other in every variety and a significant correlation could be detected between photosynthetic indices and photosynthetic pigment contents. That can explain that photosynthetic efficiency will increase with the accumulation of photosynthetic pigment contents. Besides, our result was the same as Soares's result. Their experience showed that photosynthetic pigment contents directly influenced photosynthetic efficiency (Soares et al., 2020). Based on this result, we could enhance crop photosynthesis efficiency by postponing the degradation or wreck in photosynthetic pigments.

\section{Relationship between photosynthesis and yield}

Photosynthesis was one main process to accumulate dry matter, and dry matter served as the basis in the formation of yield (Buesa et al., 2020). So photosynthesis played an important role in the formation of yield. In order to further confirm that photosynthesis was connected with yield, we measured three kernel traits, and analyzed the correlation with each other. The result confirmed the correctness of our guess and that the rising rate of photosynthesis could enhance crop yield. Based on the result, we raised another guess: whether there was a direct relationship between photosynthetic pigment contents and yield. Unexpectedly, the correlation was lower and not remarkable between photosynthetic pigment and yield. The research explained that photosynthetic pigments did not have a direct influence on yield. However, our result was different with Gutierrez et al. (2010) and Hamblin et al. (2014), and they had a contrary conclusion. Gutierrez et al. (2010) found there was a positive relationship between chlorophyll contents with grain yield, while Hamblin et al. (2014) showed that decrease in chlorophyll contents could lead to high yields.

\section{Relationship between quality and yield of broomcorn millet}

In addition to yield, qualities and nutrition of crop have also been extensively concerned. Broomcorn millet has a long history as human food and is one of the important coarse cereals. As an important crop, it is rich in carotenoids, which are good for human health. Carotenoids mainly include zeaxanthin and lutein, and play a fundamental role in human nutrition. Carotenoids can prevent various eye and cardiovascular diseases. Most likely, it can be used as an antioxidant and to regulate the immune system which makes it effective against several types of age-related diseases (Tan and Norhaizan, 2019; Bernstein and Arunkumar, 2020). As important nutrition most studies have been done on maize (Owens et al., 2014; Chang et al., 2015). From previous experience, we found JS7 had a higher yield than other varieties, but it still remains unclear whether there is a positive relationship between yield and carotenoid contents. Millet color is an important factor for people to consume and it is affected by carotenoids. So we compared five varieties in carotenoid contents and the beige of 
millet. The results showed that JS7, a high yield variety, had a middle level in the grain beige and had a maximum in CCI, but its zeaxanthin, lutein and total carotenoid content had a minimum value. Then, in order to further analyze the relationship between carotenoid contents and yield, the correlation was detected and the result showed that there was a negative correlation between them, but the negative correlation was not significant. Thus, there was no direct relationship between carotenoid contents and yield, and we may provide a new idea that the yield can be increased by keeping high carotenoid contents, or enhancing carotenoid contents but maintaining the crop high yield.

\section{Conclusions}

In summary, we found photosynthetic pigment contents were related to photosynthetic efficiency, and they had a positive relationship, which could be used in agricultural production. Nevertheless, photosynthetic pigment contents do not directly influence the crop yield, and they may adjust yield via affecting photosynthetic efficiency. Then the analysis in carotenoid contents showed that there was no direct relationship between carotenoid contents and yield, so it was possible to enhance the carotenoid contents and yield at the same time, which provides a new idea, compared with the old thought that high yield is contradictory with good qualities in crop production. Carotenoids were also correlated with the accumulation of some important quality traits, such as starch, reducing sugar and crude protein (Xu et al., 2010), which further proves that it is possible to breed high-yield and high-quality varieties in the breeding practice of high carotene type varieties. Our results need to be further confirmed by molecular biology, crossbreeding, and other methods, which can provide scientific basis and parent materials for breeding of high quality, high yield and high carotene type varieties.

Acknowledgements. This work was financially supported by Creation of New Germplasm of Characteristic Crops and Demonstration of Organic Dry Farming Technology (KY202002002), the Scientific and Technological Innovation Breeding Programs of Shanxi Agriculture University (20132-23), and Technology Innovation Fund of Shanxi Agricultural University (2017GPY01).

\section{REFERENCES}

[1] Amadou, I., Gounga, M. E., Le, G. W. (2013): Millets: Nutritional composition, some health benefits and processing-A review. - Emir. J. Food Agric 25: 501-508.

[2] Azad, M. O. K., Jeong, D. I., Adnan, M., Salitxay, T., Heo, J. W., Naznin, M. T., Lim, J. D., Cho, D. H., Park, B. J., Park, C. H. (2019): Effect of Different Processing Methods on the Accumulation of the Phenolic Compounds and Antioxidant Profile of Broomcorn Millet (Panicum miliaceum L.) Flour. - Foods 8: 230.

[3] Bernstein, P. S., Arunkumar, R. (2020): The emerging roles of the macular pigment carotenoids throughout the lifespan and in prenatal supplementation. - Journal of lipid research. DOI: 10.1194/jlr.TR120000956.

[4] Buesa, I., Miras-Avalos, J. M., Intrigliolo, D. S. (2020): Row orientation effects on potted-vines performance and water-use efficiency. - Agric. For. Meteorol. 294: 108148.

[5] Challinor, A. J., Watson, J., Lobell, D. B., Howden, S. M., Smith, D. R., Chhetri, N. (2014): A meta-analysis of crop yield under climate change and adaptation. - Nat. Clim. Change 4: 287-291. 
[6] Chang, S., Berman, J., Sheng, Y. M., Wang, Y. D., Capell, T., Shi, L., Ni, X. Z., Sandmann, G., Christou, P., Zhu, C. F. (2015): Cloning and functional characterization of the maize (Zea mays L.) carotenoid epsilon hydroxylase gene. - PloS One 10: e0128758.

[7] Diao, X. (2017): Production and genetic improvement of minor cereals in China. - The Crop Journal 5(2): 103-114.

[8] Dong, Y. M., De, X. S., Yi, Z., Chen, Y. W., Yun, J. Z., Tian, C. G. (2014): Diversity of Antioxidant Content and Its Relationship to Grain Color and Morphological Characteristics in Winter Wheat Grains. - J. Integr. Agric. 13: 1258-1267.

[9] Farre, G., Bai, C., Twyman, R. M., Capell, T., Christou, P., Zhu, C. F. (2011): Nutritious crops producing multiple carotenoids-a metabolic balancing act. - Trends Plant Sci 16: 532-540.

[10] Fu, X., Kong, W., Peng, G., Zhou, J. Y., Azam, M., Xu, C. J., Grierson, D., Chen, K. S. (2012): Plastid structure and carotenogenic gene expression in red- and white-fleshed loquat (Eriobotrya japonica) fruits. - J. Ex.B 63: 341-354.

[11] Gutierrez, M., Reynolds, M. P., Raun, W. R., Stone, M. L., Klatt, A. R. (2010): Spectral water indices for assessing yield in elite bread wheat genotypes under well-irrigated, water-stressed, and high-temperature conditions. - Crop Sci 50: 197-214.

[12] Habiyaremye, C., Matanguihan, J. B., Guedes, J. D., Ganjyal, G. M., Whiteman, M. R., Kidwell, K. K., Murphy, K. M. (2017): Proso Millet (Panicum miliaceum L.) and Its Potential for Cultivation in the Pacific Northwest, U. S. - A Review. - Front. Plant Sci. 7: 1961.

[13] Hamblin, J., Stefanova, K., Angessa, T. T. (2014): Variation in chlorophyll content per unit leaf area in spring wheat and implications for selection in segregating material. PloS One 9: e92529.

[14] Hou, S., Sun, Z., Li, Y., Wang, Y., Ling, H., Xing, G., Han, Y. H., Li, H. Y. (2017): Transcriptomic analysis, genic ssr development, and genetic diversity of proso millet (Panicum miliaceum; Poaceae). - Applications in Plant Sciences 5(7): 1600137.

[15] Huang, M. X., Wang, J., Tang, J. Z., Yu, Q., Zhang, J., Xue, Q. Y., Chang, Q., Tan, M. X. (2016): Suitability of four stomatal conductance models in agro-pastoral ecotone in North China: A case study for potato and oil sunflower. - Ying Yong Sheng Tai Xue Bao J. Appl. Ecol. 27: 3585-3592.

[16] Kumar, D., Singh, H., Raj, S., Soni, V. (2020): Chlorophyll a fluorescence kinetics of mung bean (Vigna radiata L.) grown under artificial continuous light. - Biochem. Biophys. Rep. 24: 100813-100813.

[17] Leipe, C., Long, T., Sergusheva, E. A., Wagner, M., Tarasov, P. E. (2019): Discontinuous spread of millet agriculture in eastern Asia and prehistoric population dynamics. Science Advances 5(9): eaax6225.

[18] Liu, M., Zhang, Z., Ren, G., Zhang, Q., Wang, Y., Lu, P. (2016): Evaluation of selenium and carotenoid concentrations of 200 foxtail millet accessions from China and their correlations with agronomic performance. - J. Integr. Agric. 15: 1449-1457.

[19] Lu, H., Zhang, J., Liu, K. B., Wu, N., Li, Q. (2009): Earliest domestication of common millet (Panicum miliaceum) in East Asia extended to 10,000 years ago. - P. Natl. Acad. Sci. USA 106: 7367-7372.

[20] Owens, B. F., Lipka, A. E., Magallaneslundback, M., Tiede, T., Diepenbrock, C. H., Kandianis, C. B., Kim, E., Cepela, J., Mateos, M., Buell, C. R., Buckler, E. S., Penna, D. D., Gore, M. A., Rocheford, T. (2014): A foundation for provitamin a biofortification of maize: Genome-wide association and genomic prediction models of carotenoid levels. Genetics 198: 699-716.

[21] Pinto, H., Sharwood, R. E., Tissue, D. T., Ghannoum, O. (2014): Photosynthesis of C3, C3-C4, and C4 grasses at glacial CO2. - J. Exp. Bot 13: 3669-3681.

[22] Princiotta, F. T., Loughlin, D. H. (2014): Global climate change: The quantifiable sustainability challenge. - J. Air Waste Manage 64: 979-994. 
[23] Rasool, A., Shah, W. H., Tahir, I., Alharby, H. F., Hakeem, K. R., Rehman, R. (2020): Exogenous application of selenium (Se) mitigates $\mathrm{NaCl}$ stress in proso and foxtail millets by improving their growth, physiology and biochemical parameters. - Acta Physiol. Plant 42: 116.

[24] Shi, W., Yue, L., Guo, J. H., Wang, J., Yuan, X., Dong, S., Guo, J., Guo, P. Y. (2020): Identification and evolution of $\mathrm{C}-4$ photosynthetic pathway genes in plants. - Bmc Plant Biol 20: 132.

[25] Soares, C., Pereira, R., Martins, M., Tamagnini, P., Serodio, J., Moutinho-Pereira, J., Cunha, A., Fidalgo, F. (2020): Glyphosate-dependent effects on photosynthesis of Solanum lycopersicum L.-An ecophysiological, ultrastructural and molecular approach. J. Hazard. Mater 398: 122871.

[26] Tan, B. L., Norhaizan, M. E. (2019): Carotenoids: How Effective Are They to Prevent Age-Related Diseases? - Molecules 24: 1801.

[27] Wang, Y., Long, S. P., Zhu, X. (2014): Elements required for an efficient NADP-malic enzyme type C4 photosynthesis. - Plant Physiol 164: 2231-2246.

[28] Xu, J., Hu, Q., Wang, X., Luo, J., Liu, Y., Tian, C. (2010): Changes in the Main Nutrients, Phytochemicals, and Antioxidant Activity in Yellow Corn Grain during Maturation. - Agric Food Chem 58: 5751-5756.

[29] Xue, L., Ma, J. J., Wu, J., Zhao, C., Liu, H., Liu, D., Ma, J. F. (2019): Comprehensive Evaluation on the Tolerance of Eight Crop Species to $\mathrm{CO} 2$ Leakage from Geological Storage. - Int. J. Agric. Biol. 22: 561-568.

[30] Zhang, L., Liu, R., Niu, W. (2014): Phytochemical and antiproliferative activity of proso millet. - PloS One 9: e104058.

[31] Zhou, J. Y., Sun, C. D., Zhang, L. L., Dai, X., Xu, C. J., Chen, K. S. (2010): Preferential accumulation of orange-colored carotenoids in Ponkan (Citrus reticulata) fruit peel following postharvest application of ethylene or ethephon. - Sci. Hortic-Ameterdam 126: 229-235. 